\title{
GROWTH IN THE MINIMAL INJECTIVE RESOLUTION OF A LOCAL RING
}

\author{
LARS WINTHER CHRISTENSEN, JANET STRIULI, AND OANA VELICHE
}

\begin{abstract}
Let $R$ be a commutative noetherian local ring with residue field $\mathrm{k}$ and assume that it is not Gorenstein. In the minimal injective resolution of $R$, the injective envelope $E$ of the residue field appears as a summand in every degree starting from the depth of $R$. The number of copies of $E$ in degree $i$ equals the k-vector space dimension of the cohomology module $\operatorname{Ext}_{R}^{i}(\mathrm{k}, R)$. These dimensions, known as Bass numbers, form an infinite sequence of invariants of $R$ about which little is known. We prove that it is non-decreasing and grows exponentially if $R$ is Golod, a non-trivial fiber product, or Teter, or if it has radical cube zero.
\end{abstract}

\section{INTRODUCTION}

In this paper $R$ is a commutative noetherian local ring with maximal ideal $\mathfrak{m}$. It is a pervasive theme in local algebra that properties of $R$ can be retrieved from homological invariants of the residue field $\mathrm{k}=R / \mathfrak{m}$. A prime example is vanishing of cohomology with coefficients in $\mathrm{k}$. Indeed, $R$ is regular if and only if $\operatorname{Ext}_{R}^{i}(\mathrm{k}, \mathrm{k})=0$ for all $i \gg 0$, and $R$ is Gorenstein if and only if $\operatorname{Ext}_{R}^{i}(\mathrm{k}, R)=0$ for all $i \gg 0$.

The cohomology groups $\operatorname{Ext}_{R}^{i}(\mathrm{k}, \mathrm{k})$ behave rigidly: if $R$ is singular (i.e. not regular), then they are all non-zero. In this case, one focuses on their size, captured by the sequence of invariants $\beta_{i}^{R}(\mathrm{k})=\operatorname{rank}_{\mathrm{k}} \operatorname{Ext}_{R}^{i}(\mathrm{k}, \mathrm{k})$, called the Betti numbers of $k$. Through work pioneered by Gulliksen [12, the asymptotic behavior of these Betti numbers is understood well enough to provide valuable information about singular local rings. The sequence $\left\{\beta_{i}^{R}(\mathrm{k})\right\}_{i \geqslant 0}$ is known to be non-decreasing; it is eventually constant if and only if $R$ is a hypersurface, and it has polynomial growth if and only if $R$ is a complete intersection. If $R$ is not a complete intersection, then the Betti numbers are increasing and they grow exponentially.

As shown by Foxby [8], also the cohomology groups $\operatorname{Ext}_{R}^{i}(\mathrm{k}, R)$ behave rigidly: if $R$ is not Gorenstein, then $\operatorname{Ext}_{R}^{i}(\mathrm{k}, R)$ is non-zero for all $i \geqslant \operatorname{depth} R$. Their size is captured by the Bass numbers $\mu^{i}(R)=\operatorname{rank}_{\mathrm{k}} \operatorname{Ext}_{R}^{i}(\mathrm{k}, R)$, but these invariants are not understood nearly as well as the Betti numbers $\beta_{i}^{R}(\mathrm{k})$. It is not even known if the sequence $\left\{\mu^{i}(R)\right\}_{i \geqslant \operatorname{depth} R}$ is non-decreasing. Existence of a local ring which is not Gorenstein and whose Bass numbers grow polynomially is also an open question.

Let $M$ be a finitely generated $R$-module. For $i \geqslant 0$ the $i^{\text {th }}$ Betti number of $M$ is defined as $\beta_{i}^{R}(M)=\operatorname{rank}_{\mathrm{k}} \operatorname{Ext}_{R}^{i}(M, \mathrm{k})$. Let $\widehat{R}$ denote the m-adic completion of $R$.

Date: 6 July 2009

2000 Mathematics Subject Classification. Primary 13D02; secondary 13D07, 13H10.

Key words and phrases. Bass number, Betti number, minimal free resolution, minimal injective resolution.

This work started while L.W.C. visited the University of Nebraska-Lincoln, partly supported by a grant from the Carlsberg Foundation. J.S. was supported by NSF grant DMS 0201904 . 
Foxby [9] shows that there is a finitely generated $\widehat{R}$-module $\Omega$ such that one has

$$
\mu^{\operatorname{dim} R+i}(R)=\beta_{i}^{\widehat{R}}(\Omega) \quad \text { for all } i \geqslant 0 .
$$

Thus, the non-zero Bass numbers of $R$, except the first $c:=\operatorname{dim} R-\operatorname{depth} R$, can be realized as the Betti numbers of a module. In particular, the sequence $\left\{\mu^{i}(R)\right\}_{i \geqslant d}$ has at most exponential growth. If $R$ is Cohen-Macaulay, then $c=0$ and $\Omega$ is the canonical module for $\widehat{R}$. Jorgensen and Leuschke [15] take this approach to prove that the sequence $\left\{\mu^{i}(R)\right\}_{i \geqslant \operatorname{depth} R}$ grows exponentially and is eventually increasing for certain families of Cohen-Macaulay rings. Their work was motivated by a question of Huneke about the asymptotic behavior of these numbers; however, they are naturally lead to raise a question about the initial behavior [15, 2.6]:

(1.1) Question. Let $R$ be a Cohen-Macaulay local ring of depth $d$. If the inequality $\mu^{d+1}(R) \leqslant \mu^{d}(R)$ holds, is then $R$ Gorenstein?

There is already a history in local algebra for studying the initial behavior of Bass numbers - in particular, the number $\mu^{\operatorname{dim} R}(R)$ which is called the type of $R$. Bass [7] characterized Gorenstein rings as Cohen-Macaulay rings of type 1. Later, Foxby 9$]$ and Roberts [22] proved that any local ring with $\mu^{\operatorname{dim} R}(R)=1$ is Gorenstein. This confirmed a conjecture of Vasconcelos [27].

$$
* * *
$$

In this paper we study the initial, local, and asymptotic behavior of Bass numbers for certain families of local rings; by local behavior we mean comparison of a few consecutive numbers. While the realization of Bass numbers as Betti numbers (1.0.1) remains important for our work, it provides no information about the initial behavior of the Bass numbers of a ring that is not Cohen-Macaulay. We employ a bag of ad hoc methods to deal with this situation.

The embedding dimension of $R$, denoted edim $R$, is the minimal number of generators of the maximal ideal. Question (1.1) is answered affirmatively in [15] when edim $R$ is $d+2$, where $d$ denotes the depth of $R$. This is the first interesting, case inasmuch as $R$ is a hypersurface if $\operatorname{edim} R \leqslant d+1$. We improve the result from [15] by computing the Bass numbers of these rings in closed form; see (2.8). The conclusion is strong: if $R$ is not Gorenstein, then there are (in)equalities

$$
\mu^{d+1}(R)=\mu^{d}(R)+1 \quad \text { and } \mu^{i+1}(R) \geqslant 2 \mu^{i}(R) \text { for all } i \geqslant d+1 .
$$

Similar detailed information is obtained for other rings. The Main Theorem below extracts the overall conclusion that applies to several families of rings. For the Cohen-Macaulay rings among them, it answers Question (1.1) affirmatively.

(1.2) Main Theorem. Let $(R, \mathfrak{m}, \mathrm{k})$ be a local ring of embedding dimension $e$ at least 2 and depth $d$. Assume that $R$ satisfies one of the following conditions:

(1) $R$ is Golod and it has $e-d>2$ or $\mu^{d}(R)>1$.

(2) $R$ is the fiber product of two local rings (both different from $\mathrm{k}$ ) and not Golod.

(3) $R$ is artinian with $\operatorname{Soc} R \nsubseteq \mathfrak{m}^{2}$.

(4) $R$ is not Gorenstein and $\mathfrak{m}^{3}=0$.

(5) $R$ is Teter; that is, $R \cong Q / \operatorname{Soc} Q$ where $Q$ is artinian and Gorenstein.

Then the sequence of Bass numbers $\left\{\mu^{i}(R)\right\}_{i \geqslant d}$ is increasing and has exponential growth; if $R$ satisfies (1), (3), (4), or (5), then the growth is termwise exponential. 
Notice that the assumption edim $R \geqslant 2$ only excludes hypersurface. A sequence $\left\{a_{i}\right\}_{i \geqslant 0}$ is said to have exponential growth if there exists a real number $A>1$ such that $a_{i} \geqslant A^{i}$ for all $i \gg 0$, and the growth it said to be termwise exponential of rate $A$ if there exists a real number $A>1$ such that $a_{i+1} \geqslant A a_{i}$ for all $i \gg 0$.

Golod rings and fiber products are, in general, far from being Gorenstein, so one could expect their Bass numbers grow rapidly. Teter rings have been called "almost Gorenstein" 14, but they still differ significantly from Gorenstein rings, and some of them are even Golod. In this perspective, (4) is the most surprising part of the Main Theorem, as there is empirical evidence in [1] and other works that rings with $\mathfrak{m}^{3}=0$ are excellent grounds for testing homological questions in local algebra.

Based on the Main Theorem - and Example (1.4) below, which shows that two consecutive Bass numbers can be equal and non-zero - we extend and explicitly state the question that motivated Jorgensen and Leuschke's work [15]:

(1.3) Question. Let $R$ be a local ring of depth $d$ and assume that it is not Gorenstein. Does the sequence of Bass numbers $\left\{\mu^{i}(R)\right\}_{i \geqslant d}$ then have exponential growth, and is it non-decreasing? Is it eventually increasing, and if so, from which step?

Our work towards the Main Theorem started from two explicit computations of Bass numbers. We discovered (1.4) while computing examples with the aid of Macaulay 2 [11; it can also be deduced from work of Wiebe [28].

(1.4) Example. Let $\mathrm{F}$ be a field. The first few Bass numbers of the local ring $\mathrm{F} \llbracket x, y \rrbracket /\left(x^{2}, x y\right)$ are $1,2,2,4,6,10$.

This ring is not Cohen-Macaulay, so the example says nothing about Question (1.1); it merely frames it. The ring is the archetype of the exceptional case in part (11) of the Main Theorem. We show in Proposition (2.9) that the Bass numbers of such rings (Golod with $e=d+2$ and $\mu^{d}(R)=1$ ) are given by the Fibonacci numbers as follows: $\mu^{d+i}(R)=2 F_{i}$ for all $i \geqslant 1$. In particular, the sequence $\left\{\mu^{i}(R)\right\}_{i \geqslant d}$ is non-decreasing with termwise exponential growth, and it increases from the third step.

Another simple example [5, (10.8.2)] provides a textbook illustration of termwise exponential growth. The Main Theorem generalizes it in several directions.

(1.5) Example. For a local ring $(R, \mathfrak{m})$ with $\mathfrak{m}^{2}=0$ and embedding dimension $e \geqslant 2$, the Bass numbers are $\mu^{0}(R)=e$ and $\mu^{i}(R)=e^{i-1}\left(e^{2}-1\right)$ for all $i \geqslant 1$.

These rings are in the intersection of the five families in the Main Theorem. Indeed, they are Golod and they trivially satisfy $\mathfrak{m}^{3}=0$, so they belong to (11) and (4). Since they have $\operatorname{Soc} R=\mathfrak{m}$, they belong to (31) and, as will be explained in (3.7), also to (2). Finally, it is a result of Teter [26] that a local ring with $\mathfrak{m}^{2}=0$ is Teter.

The organization of the paper follows the agenda set by the Main Theorem. The Appendix has results on local and asymptotic behavior of Betti numbers for modules over artinian rings. These are used in the proofs of parts (3) and (4) of the Main Theorem, which make heavy use of the realization of Bass numbers as Betti numbers (1.0.1). A curious upshot - an immediate consequence of Lemma (A.1) - is a reformulation of Bass' characterization of Gorenstein rings: 
(1.6) Characterization. If $R$ is a Cohen-Macaulay local ring and $\mu^{n}(R)=1$ for some $n \geqslant 0$, then $R$ is Gorenstein of dimension $n$.

Example (1.4) shows that the Cohen-Macaulay hypothesis is necessary in (1.6), but it would be interesting to know if there is a similar reformulation of the result of Foxby [9] and Roberts [22]. The best one can hope for is a positive answer to:

(1.7) Question. Let $R$ be a local ring. If $\mu^{n}(R)=1$ for some $n \geqslant \operatorname{dim} R$, is then $R$ Gorenstein of dimension $n$ ?

If $R$ is an integral domain, then an affirmative answer is already contained in 22.

\section{GOLOD RINGS}

The central result of this section, Theorem (2.5), is part (1) of the Main Theorem. Throughout the section, $d$ denotes the depth of $R$ and $e$ its embedding dimension.

We use the standard notation for Koszul homology: given a sequence $\boldsymbol{y}$ of elements in the maximal ideal $\mathfrak{m}$ and an $R$-module $M$, the $i^{\text {th }}$ homology module of the Koszul complex $\mathrm{K}(\boldsymbol{y}) \otimes_{R} M$ is denoted $\mathrm{H}_{i}(\boldsymbol{y} ; M)$. Moreover, the notation $\mathrm{H}_{i}(\boldsymbol{y} ; R)$ is abbreviated $\mathrm{H}_{i}(\boldsymbol{y})$; see also [24, IV.A.§1].

(2.1) Golod rings are the local rings for which the Betti numbers of the residue field have extremal growth; see 2, sec. 5]. All hypersurface rings are Golod, and a Golod ring is Gorenstein if and only if it is a hypersurface; see 2, rmk. after prop. 5.2.5]. The codimension of $R$ is defined $\operatorname{as} \operatorname{codim} R=\operatorname{edim} R-\operatorname{dim} R$. Every ring of codimension at most 1 is Golod; see 2, prop. 5.2.5]. Thus, the ring in Example (1.4) is Golod. So is the ring in Example (1.5), but for a different reason; see [2, prop. 5.3.4.(1)].

Here we use a characterization of Golod rings in terms of Bass numbers. The Bass numbers of $R$ are encoded into a formal power series,

$$
\mathrm{I}_{R}(t)=\sum_{i=0}^{\infty} \mu^{i}(R) t^{i}
$$

called the Bass series of $R$.

Assume that $R$ is singular, and let $\boldsymbol{x}$ be a minimal system of generators for $\mathfrak{m}$. Avramov and Lescot [4, (0.2)] prove that there is a coefficient-wise inequality

$$
\mathrm{I}_{R}(t) \preccurlyeq \frac{\sum_{i=0}^{e-1} \operatorname{rank}_{\mathrm{k}} \mathrm{H}_{e-i}(\boldsymbol{x}) t^{i}-t^{e+1}}{1-\sum_{i=1}^{e} \operatorname{rank}_{\mathrm{k}} \mathrm{H}_{i}(\boldsymbol{x}) t^{i+1}},
$$

where equality holds if and only if $R$ is Golod.

A crucial step in the proof of Theorem (2.5) is a reduction of (2.1.1), which comes about because -1 is a common root of the numerator and the denominator. One can deduce this from the work of Avramov, Iyengar, and Miller [3]. In Lemma (2.3) we provide a direct argument; first we split the coefficients $\operatorname{rank}_{\mathrm{k}} \mathrm{H}_{j}(\boldsymbol{x})$ in (2.1.1):

(2.2) Lemma. Let $R$ be singular, and let $\boldsymbol{x}=x_{1}, \ldots, x_{e}$ be a minimal system of generators of $\mathfrak{m}$ such that $\boldsymbol{x}^{\prime}=x_{1}, \ldots, x_{e-1}$ generates an $\mathfrak{m}$-primary ideal. For every integer $i$ the Koszul homology module $\mathrm{H}_{0}\left(x_{e} ; \mathrm{H}_{i}\left(\boldsymbol{x}^{\prime}\right)\right)$ is a finite dimensional $\mathrm{k}$-vector space, and it is non-zero if and only if $i \in\{0, \ldots, e-d-1\}$. Moreover, for every $i \geqslant 0$ there is an equality

$$
\operatorname{rank}_{\mathbf{k}} \mathrm{H}_{i}(\boldsymbol{x})=\operatorname{rank}_{\mathbf{k}} \mathrm{H}_{0}\left(x_{e} ; \mathrm{H}_{i}\left(\boldsymbol{x}^{\prime}\right)\right)+\operatorname{rank}_{\mathbf{k}} \mathrm{H}_{0}\left(x_{e} ; \mathrm{H}_{i-1}\left(\boldsymbol{x}^{\prime}\right)\right) .
$$


Proof. Since $\boldsymbol{x}^{\prime}$ generates an $\boldsymbol{m}$-primary ideal, the homology module $\mathrm{H}_{i}\left(\boldsymbol{x}^{\prime}\right)$ has finite length for every $i$, and by depth sensitivity and rigidity it is non-zero if and only if $0 \leqslant i \leqslant e-d-1$; see [24, prop. IV.3]. For every $i$ and $j$ the module $\mathrm{H}_{j}\left(x_{e} ; \mathrm{H}_{i}\left(\boldsymbol{x}^{\prime}\right)\right)$ is annihilated by $\mathfrak{m}$ and hence it is a finite dimensional k-vector space. By Nakayama's lemma, $\mathrm{H}_{0}\left(x_{e} ; \mathrm{H}_{i}\left(\boldsymbol{x}^{\prime}\right)\right)$ is non-zero if and only if $\mathrm{H}_{i}\left(\boldsymbol{x}^{\prime}\right)$ is so.

For every $i$, there is a short exact sequence of finite dimensional k-vector spaces

$$
0 \rightarrow \mathrm{H}_{0}\left(x_{e}, \mathrm{H}_{i}\left(\boldsymbol{x}^{\prime}\right)\right) \rightarrow \mathrm{H}_{i}(\boldsymbol{x}) \rightarrow \mathrm{H}_{1}\left(x_{e}, \mathrm{H}_{i-1}\left(\boldsymbol{x}^{\prime}\right)\right) \rightarrow 0 ;
$$

see [24, prop. IV.1]. It yields

$$
\operatorname{rank}_{\mathbf{k}} \mathrm{H}_{i}(\boldsymbol{x})=\operatorname{rank}_{\mathbf{k}} \mathrm{H}_{0}\left(x_{e} ; \mathrm{H}_{i}\left(\boldsymbol{x}^{\prime}\right)\right)+\operatorname{rank}_{\mathbf{k}} \mathrm{H}_{1}\left(x_{e} ; \mathrm{H}_{i-1}\left(\boldsymbol{x}^{\prime}\right)\right) .
$$

To finish the proof we need to verify the equality

$$
\operatorname{rank}_{\mathbf{k}} \mathrm{H}_{1}\left(x_{e}, \mathrm{H}_{i-1}\left(\boldsymbol{x}^{\prime}\right)\right)=\operatorname{rank}_{\mathrm{k}} \mathrm{H}_{0}\left(x_{e}, \mathrm{H}_{i-1}\left(\boldsymbol{x}^{\prime}\right)\right) \text {. }
$$

It follows from a length count in the exact sequence

$$
0 \rightarrow \mathrm{H}_{1}\left(x_{e}, \mathrm{H}_{i-1}\left(\boldsymbol{x}^{\prime}\right)\right) \rightarrow \mathrm{H}_{i-1}\left(\boldsymbol{x}^{\prime}\right) \stackrel{x_{e}}{\longrightarrow} \mathrm{H}_{i-1}\left(\boldsymbol{x}^{\prime}\right) \rightarrow \mathrm{H}_{0}\left(x_{e}, \mathrm{H}_{i-1}\left(\boldsymbol{x}^{\prime}\right)\right) \rightarrow 0 \text {. }
$$

(2.3) Lemma. Let $R$ be singular and let $\boldsymbol{x}=x_{1}, \ldots, x_{e}$ be a minimal system of generators of $\mathfrak{m}$ such that $\boldsymbol{x}^{\prime}=x_{1}, \ldots, x_{e-1}$ generates an $\mathfrak{m}$-primary ideal. For $i \geqslant 0$ set $h_{i}=\operatorname{rank}_{\mathrm{k}} \mathrm{H}_{0}\left(x_{e}, \mathrm{H}_{i}\left(\boldsymbol{x}^{\prime}\right)\right)$. Then there is a coefficient-wise inequality

$$
\mathrm{I}_{R}(t) \preccurlyeq \frac{\sum_{i=0}^{e-d-1} h_{e-d-1-i} t^{d+i}-t^{e}}{1-\sum_{i=0}^{e-d-1} h_{i} t^{i+1}},
$$

and equality holds if and only if $R$ is Golod.

Proof. Since $R$ is singular, we have $e-d \geqslant 1$. For $i \geqslant 0$ set $c_{i}=\operatorname{rank}_{\mathrm{k}} \mathrm{H}_{i}(\boldsymbol{x})$, then (2.1.1) takes the form

$$
\mathrm{I}_{R}(t) \preccurlyeq \frac{\sum_{i=0}^{e-1} c_{e-i} t^{i}-t^{e+1}}{1-\sum_{i=1}^{e} c_{i} t^{i+1}},
$$

and equality holds if and only if $R$ is Golod. We first verify that -1 is a root of both the numerator and denominator. Indeed, by Lemma (2.2) there are equalities $c_{e-i}=h_{e-i}+h_{e-i-1}$; in particular, $h_{0}=c_{0}=1$. Now we have

$$
\begin{aligned}
\sum_{i=0}^{e-1} c_{e-i}(-1)^{i}-(-1)^{e+1} & =\sum_{i=d+1}^{e-1}\left(h_{e-i}+h_{e-i-1}\right)(-1)^{i}-(-1)^{e+1} \\
& =h_{0}(-1)^{e-1}-(-1)^{e+1}=0
\end{aligned}
$$

and

$$
1-\sum_{i=1}^{e} c_{i}(-1)^{i+1}=1-\sum_{i=1}^{e-d}\left(h_{i}+h_{i-1}\right)(-1)^{i+1}=1-h_{0}(-1)^{2}=0 .
$$

Cancellation of the common factor $1+t$ gives the equality

$$
\frac{\sum_{i=0}^{e-1} c_{e-i} t^{i}-t^{e+1}}{1-\sum_{i=1}^{e} c_{i} t^{i+1}}=\frac{\sum_{i=0}^{e-d-1} h_{e-d-1-i} t^{d+i}-t^{e}}{1-\sum_{i=0}^{e-d-1} h_{i} t^{i+1}} .
$$

(2.4) Observation. Let $R$ be Golod and assume it is not Gorenstein - that is, not a hypersurface - then one has $e-d \geqslant 2$; see [2, 5.1]. Let $h_{i}$ for $i \geqslant 0$ be as defined in Lemma (2.3). As $h_{0}=1$ the Bass series of $R$ takes the form

$$
\mathrm{I}_{R}(t)=\frac{\sum_{i=0}^{e-d-2} h_{e-d-1-i} t^{d+i}+t^{e-1}-t^{e}}{1-t-\sum_{i=1}^{e-d-1} h_{i} t^{i+1}} .
$$


Set $\mu^{i}=\mu^{i}(R)$ for $i \geqslant 0$. It is straightforward to deduce the next equalities from (2.4.1); one can also extract them from the proof of [4, (0.2)].

$$
\begin{aligned}
\mu^{d} & =h_{e-d-1} \\
\mu^{e} & =\mu^{e-1}+\sum_{i=0}^{e-d-2} \mu^{d+i} h_{e-d-1-i}-1, \quad \text { and } \\
\mu^{e+n} & =\mu^{e+n-1}+\sum_{i=n}^{n+e-d-2} \mu^{d+i} h_{e-d-1+n-i} \text { for } n \geqslant 1 .
\end{aligned}
$$

The expression for the rate of growth $A$ in the next theorem is inspired by Peeva's proof of [21, prop. 3]; see also [2, thm. 5.3.3.(5)]. In view of (1.0.1) it follows from the latter result that the Bass sequence for a Golod ring $R$ with $e-d \geqslant 2$ has termwise exponential growth. The next theorem and Proposition (2.9) explains the initial behavior of these Bass sequences.

(2.5) Theorem. Let $R$ be Golod of depth $d$ and embedding dimension $e$. If one of the inequalities $e-d>2$ or $\mu^{d}(R)>1$ holds, then the sequence $\left\{\mu^{i}(R)\right\}_{i \geqslant d}$ is increasing, and it has termwise exponential growth of rate

$$
A=\min \left\{\frac{\mu^{d+1}(R)}{\mu^{d}(R)}, \frac{\mu^{d+2}(R)}{\mu^{d+1}(R)}, \ldots, \frac{\mu^{e}(R)}{\mu^{e-1}(R)}\right\}>1 .
$$

(2.6) Remark. In the exceptional case with $e-d=2$ and $\mu^{d}(R)=1$ 丹, the Bass numbers of $R$ are given by $\mu^{d+i}(R)=2 F_{i}$ for $i \geqslant 1$, where $F_{i}$ is the $i^{\text {th }}$ Fibonacci number. In particular the sequence $\left\{\mu^{i}(R)\right\}_{i \geqslant d}$ is non-decreasing and it has termwise exponential growth; see Proposition (2.9) and the remark that follows it.

Proof of Theorem (2.5) . It follows from the assumptions on $R$ that it is not a hypersurface, so we have $e-d \geqslant 2$. For $i \geqslant 0$ set $\mu^{i}=\mu^{i}(R)$ and adopt the notation from Lemma (2.3). There is a coefficient-wise inequality

$$
\begin{aligned}
(1-t) \mathrm{I}_{R}(t) & \succcurlyeq\left(1-t-\sum_{i=1}^{e-d-1} h_{i} t^{i+1}\right) \mathrm{I}_{R}(t) \\
& =\sum_{i=0}^{e-d-2} h_{e-d-1-i} t^{d+i}+t^{e-1}-t^{e},
\end{aligned}
$$

where the equality follows from (2.4.1). In particular, there are the following inequalities among the coefficients of $\mathrm{I}_{R}(t)$ :

$$
\mu^{d}<\mu^{d+1}<\cdots<\mu^{e-1} .
$$

Moreover, at least one of the inequalities $\mu^{d} \geqslant 2$ or $e-d-2 \geqslant 1$ holds, so (2.4.3) yields $\mu^{e-1}<\mu^{e}$ and, therefore, $A>1$. By recursion it now follows from (2.4.4) and (2.4.3) that $\mu^{e+n} \geqslant A \mu^{e+n-1}$ for every $n \geqslant 1$.

(2.7) Corollary. If $R$ is Golod of codimension at least 2, then the sequence of Bass numbers $\left\{\mu^{i}(R)\right\}_{i \geqslant d}$ is increasing and has termwise exponential growth.

\footnotetext{
* Let $\mathrm{F}$ be a field. The ring $R=\mathrm{F} \llbracket x, y \rrbracket /\left(x^{2}, x y\right)$ is an example. Indeed, it has Krull dimension 1 and depth 0 , so it is Golod by [2, prop. 5.2.5], and $\operatorname{Hom}_{R}(\mathrm{k}, R)$ is generated by $x$, so $\mu^{0}(R)=1$.
} 
Proof. By assumption there is an inequality $e-d \geqslant 2$; in particular $R$ is not a hypersurface and hence not Gorenstein. If equality holds, then $R$ is CohenMacaulay, and then one has $\mu^{d}(R)>1$ by [7, thm. 6.3]. The statement now follows from Theorem (2.5).

(2.8) Remark. This corollary covers Cohen-Macaulay rings of codimension 2 that are not Gorenstein. Indeed, such rings are Golod by [23]; see also [2, prop. 5.3.4]. Also the next proposition applies to Cohen-Macaulay rings of codimension 2.

(2.9) Proposition. Let $R$ be of depth $d$ and embedding dimension $e=d+2$; set $r=\mu^{d}(R)$. If $R$ is not a complete intersection, then there is an equality

$$
\mathrm{I}_{R}(t)=t^{d} \frac{r+t-t^{2}}{1-t-r t^{2}}
$$

That is, the Bass numbers of $R$ are

$$
\mu^{d+i}(R)= \begin{cases}0 & \text { for } i<0 \\ r & \text { for } i=0 \\ r+1 & \text { for } i=1 \\ r(r+1) & \text { for } i=2 \\ 2 r(r+1) & \text { for } i=3 \\ \mu^{d+i-1}+r \mu^{d+i-2} & \text { for } i \geqslant 4\end{cases}
$$

Set $\delta=\sqrt{1+4 r}$; for $i \geqslant 3$ the expression for $\mu^{d+i}(R)$ in closed form is then

$$
\mu^{d+i}(R)=\frac{r+1}{2 \delta}\left((2 r-1+\delta)\left(\frac{1+\delta}{2}\right)^{i-1}+(1-2 r+\delta)\left(\frac{1-\delta}{2}\right)^{i-1}\right) .
$$

Proof. By 23. $R$ is Golod; see also [2, prop. 5.3.4]. The expression for the Bass series, therefore, follows from (2.4.1). For $i \geqslant 0$ set $\mu^{i}=\mu^{i}(R)$. A straightforward computation yields the expressions for $\mu^{d+1}$ and $\mu^{d+2}$, and (2.4.4) yields the recurrence relation

$$
\mu^{d+i}=\mu^{d+i-1}+r \mu^{d+i-2} \quad \text { for } i \geqslant 3 .
$$

The corresponding matrix $\left(\begin{array}{ll}0 & 1 \\ r & 1\end{array}\right)$ is diagonalizable with eigenvalues $\frac{1}{2}(1 \pm \sqrt{1+4 r})$, and the expression for $\mu^{d+i}$ in closed form follows.

(2.10) Remark. Let $R$ be as in Proposition (2.9). If $r=\mu^{d}(R)$ is 1 , then one has $\mu^{d+1}(R)=2=\mu^{d+2}(R)$, and the recurrence relation yields $\mu^{d+i}(R)=2 F_{i}$ for $i \geqslant 1$, where $F_{i}$ is the $i^{\text {th }}$ Fibonacci number.

If $r \geqslant 2$, then the recurrence relation and the equality $\mu^{d+3}(R)=2 \mu^{d+2}(R)$ immediately yield $\mu^{i+1}(R) \geqslant 2 \mu^{i}(R)$ for every $i \geqslant d+3$, and equality holds if an only if $r=2$. Thus, if $r=2$, then one has $\mu^{d+i}(R)=3\left(2^{i-1}\right)$ for all $i \geqslant 1$.

\section{Fiber PRODUCT RINGS}

In this section, $S$ and $T$ are local rings with the same residue field $\mathrm{k}$ and both different from $\mathrm{k}$. The fiber product $S \times_{\mathrm{k}} T$ is a local ring with residue field k and embedding dimension $e=\operatorname{edim} S+\operatorname{edim} T$. We denote its depth by $d$.

We start by observing a few fiber product rings that fail to have increasing Bass numbers, because they are either hypersurfaces or of the type considered in 
Proposition (2.9). The main result of the section-Theorem (3.4) - is that they are the only (non-trivial) ones. This will establish part (2) of the Main Theorem.

(3.1) Let $M$ be a finitely generated $R$-module. Recall that the Poincaré series of $M$ is the formal power series

$$
\mathrm{P}_{M}^{R}(t)=\sum_{i=0}^{\infty} \beta_{i}^{R}(M) t^{i}
$$

The Poincaré series of k as an $S \times{ }_{\mathrm{k}} T$-module was first computed by Kostrikin and Shafarevich [16]:

$$
\frac{1}{\mathrm{P}_{\mathrm{k}}^{S \times{ }_{\mathrm{k}} T}(t)}=\frac{1}{\mathrm{P}_{\mathrm{k}}^{S}(t)}+\frac{1}{\mathrm{P}_{\mathrm{k}}^{T}(t)}-1 .
$$

Lescot computes the quotient of the Bass series and the Poincaré series of $S \times{ }_{\mathrm{k}} T$ in [17, thm. 3.1]. For later reference we record some details from this work. If $S$ and $T$ are both singular, then one has

$$
\frac{\mathrm{I}_{S \times_{\mathrm{k}} T}(t)}{\mathrm{P}_{\mathrm{k}}^{S} \times_{\mathrm{k}} T(t)}=t+\frac{\mathrm{I}_{S}(t)}{\mathrm{P}_{\mathrm{k}}^{S}(t)}+\frac{\mathrm{I}_{T}(t)}{\mathrm{P}_{\mathrm{k}}^{T}(t)} .
$$

If $S$ is singular and $T$ is regular of dimension $n$, then the formula is

$$
\frac{\mathrm{I}_{S \times_{\mathrm{k}} T}(t)}{\mathrm{P}_{\mathrm{k}}^{S \times_{k} T}(t)}=t+\frac{\mathrm{I}_{S}(t)}{\mathrm{P}_{\mathrm{k}}^{S}(t)}-\frac{t^{n+1}}{(1+t)^{n}}
$$

If $S$ and $T$ are regular of dimension $m$ and $n$, then one has

$$
\frac{\mathrm{I}_{S \times_{\mathrm{k}} T}(t)}{\mathrm{P}_{\mathrm{k}}^{S \times_{\mathrm{k}} T}(t)}=t-\frac{t^{m+1}}{(1+t)^{m}}-\frac{t^{n+1}}{(1+t)^{n}} .
$$

The order of a power series $\sum_{i=0}^{\infty} v_{i} t^{i}$ is the number $\min \left\{i \geqslant 0 \mid v_{i} \neq 0\right\}$. Note that the order of the Bass series $\mathrm{I}_{R}(t)$ is equal to depth $R$.

(3.2) Remark. It follows from (3.1.2)-(3.1.4) that the depth of $S \times_{\mathrm{k}} T$ is at most 1, and that it is 0 if either $S$ or $T$ has depth 0 . That is, one has

$$
d=\min \{\operatorname{depth} S, \operatorname{depth} T, 1\} .
$$

(3.3) Observation. Let $S$ be a 1-dimensional regular ring. If also $T$ is regular of dimension 1, then one has $e=2$ and $d=1$ by (3.2.1), so $S \times_{\mathrm{k}} T$ is a hypersurface. If $T$ is either a 0 -dimensional hypersurface or a 2-dimensional regular ring, then one has $e-d=2$ by (3.2.1). Moreover, $S \times{ }_{\mathrm{k}} T$ is Golod as both $S$ and $T$ are Golod; see [18, thm. 4.1]. Finally, it follows from (3.1.3) and (3.1.4) that $\mu^{d}\left(S \times_{\mathrm{k}} T\right)$ is 1 in either case, and then one has $\mu^{d+1}\left(S \times_{\mathrm{k}} T\right)=2=\mu^{d+2}\left(S \times_{\mathrm{k}} T\right)$ as worked out in Remark (2.10).

(3.4) Theorem. Let $S$ and $T$ be local rings with common residue field $\mathrm{k}$ and assume that both $S$ and $T$ are different from k. If the fiber product ring $S \times_{k} T$ is not one of the three types from (3.3), then the sequence of Bass numbers $\left\{\mu^{i}\left(S \times_{\mathrm{k}} T\right)\right\}_{i \geqslant d}$ is increasing and has exponential growth.

Notice that part (2) of the Main Theorem, stated in the Introduction, follows from Theorem (3.4), as the rings in Observation (3.3) are Golod. 
For the proof of Theorem (3.4) we need some terminology and a technical lemma. A power series $\sum_{i=0}^{\infty} v_{i} t^{i}$ of order $n$ is said to have non-negative (or positive) coefficients if $v_{i} \geqslant 0$ (or $v_{i}>0$ ) for all $i \geqslant n$; it has non-decreasing (or increasing) coefficients if $v_{i+1} \geqslant v_{i}\left(\right.$ or $\left.v_{i+1}>v_{i}\right)$ for all $i \geqslant n$.

(3.5) Lemma. Let $\sum_{i=0}^{\infty} c_{i} t^{i}$ be a formal power series with increasing coefficients and assume $c_{0}>1$. Then the following hold:

(a) The coefficients of the power series

$$
V(t)=\sum_{i=0}^{\infty} v_{i} t^{i}:=\frac{1}{1-t^{2} \sum_{i=0}^{\infty} c_{i} t^{i}}
$$

grow exponentially and satisfy:

$$
v_{0}=1, \quad v_{1}=0, \quad v_{2}=c_{0}, \quad \text { and } v_{i+1}>v_{i} \text { for all } i \geqslant 2 .
$$

(b) Let $W(t)=\sum_{i=0}^{\infty} w_{i} t^{i}$ be a power series of order 0 and assume that the series $\left(1-t+t^{2}\right) W(t)$ has non-negative coefficients. Then the series $(1-t) V(t) W(t)$ has order 0 and positive coefficients in each degree except, possibly, in degree 1 where the coefficient is $w_{1}-w_{0} \geqslant 0$.

(3.6) Remark. If $R$ has positive embedding dimension, then the Poincaré series $\mathrm{P}_{\mathrm{k}}^{R}(t)$ satisfies the condition on $W(t)$ in Lemma (3.5) (b). Indeed, $\mathrm{P}_{\mathrm{k}}^{R}(t)$ is either a power series with non-decreasing coefficients or the polynomial $(1+t)^{n}$ for some $n \geqslant 1$. In the first case the claim is obvious, and in the second case it follows from the inequality $\left(\begin{array}{c}n \\ i\end{array}\right) \leqslant\left(\begin{array}{c}n \\ i-1\end{array}\right)+\left(\begin{array}{c}n \\ i+1\end{array}\right)$, which holds for all integers $i$.

Proof of Lemma (3.5) . (a): The equality $\left(1-t^{2} \sum_{i=0}^{\infty} c_{i} t^{i}\right)\left(\sum_{i=0}^{\infty} v_{i} t^{i}\right)=1$ immediately yields $v_{0}=1$ and $v_{1}=0$, and it yields $v_{2}-c_{0} v_{0}=0$ whence $v_{2}=c_{0}$. For $i \geqslant 3$ it yields $v_{i}-c_{i-2}-\sum_{j=0}^{i-3} c_{j} v_{i-2-j}=0$, and it follows by recursion that the coefficients $v_{i}$ are positive. Now the desired inequalities

$$
v_{i+1}=c_{i-1}+\sum_{j=0}^{i-2} c_{j} v_{i-1-j}>c_{i-2}+\sum_{j=0}^{i-3} c_{j} v_{i-2-j}=v_{i} \quad \text { for } i \geqslant 2
$$

follow as the sequence $\left\{c_{i}\right\}_{i \geqslant 0}$ is increasing by assumption. Finally, the expression for $v_{i}$ yields an inequality $v_{i+2}>c_{0} v_{i}$ for each $i \geqslant 2$. In particular, we have $v_{2 j}>c_{0}^{j}$ and $v_{2 j-1}>c_{0}^{j-1} c_{1}$ for $j \geqslant 2$. As $c_{1}>c_{0}>1$ we now have $v_{i}>{\sqrt{c_{0}}}^{i}$ for $i \geqslant 3$, so the sequence $\left\{v_{i}\right\}_{i \geqslant 0}$ has exponential growth.

(b): The first equality in the computation below holds as $v_{1}=0$.

$$
\begin{aligned}
(1-t) V(t) W(t) & =(1-t)\left(1+\sum_{i=2}^{\infty} v_{i} t^{i}\right) W(t) \\
& =(1-t) W(t)+t^{2} W(t)(1-t) \sum_{i=2}^{\infty} v_{i} t^{i-2} \\
& =\left(1-t+t^{2}\right) W(t)+t^{2} W(t)\left((1-t) \sum_{i=2}^{\infty} v_{i} t^{i-2}-1\right)
\end{aligned}
$$

By the assumptions on $W(t)$, the first summand in the last line of the display, $\left(1-t+t^{2}\right) W(t)$, has order 0 and non-negative coefficients; the coefficient in degree 1 is $w_{1}-w_{0}$. It follows from the inequality $v_{2}>1$ that the second summand has order 2 , and it has positive coefficients as $v_{i+1}>v_{i}$ for all $i \geqslant 2$. 
Proof of Theorem (3.4). Set $R=S \times_{\mathrm{k}} T$. Assume first that $R$ is Golod; then both $S$ and $T$ are Golod by [18, thm. 4.1]. From (3.2.1) and the equality $e=$ $\operatorname{edim} S+\operatorname{edim} T$, it is straightforward to verify that the (in)equalities $e-d \leqslant 2$ and $\mu^{d}(R)=1$ hold exactly when $R$ is one of the three types of rings from (3.3). If $e-d>2$ or $\mu^{d}(R)>1$, then it is proved in Theorem (2.5) that the sequence $\left\{\mu^{i}(R)\right\}_{i \geqslant d}$ is increasing and has exponential growth.

Assume now that $R$ is not Golod. Without loss of generality we may assume that $S$ is not a hypersurface; cf. [18, thm. 4.1]. Rewrite (3.1.1) as

$$
\mathrm{P}_{\mathrm{k}}^{R}(t)=\frac{\mathrm{P}_{\mathrm{k}}^{S}(t) \mathrm{P}_{\mathrm{k}}^{T}(t)}{1-\left(\mathrm{P}_{\mathrm{k}}^{S}(t)-1\right)\left(\mathrm{P}_{\mathrm{k}}^{T}(t)-1\right)} .
$$

Combine it with (3.1.2) to obtain

$$
\mathrm{I}_{R}(t)=\frac{\mathrm{I}_{S}(t) \mathrm{P}_{\mathrm{k}}^{T}(t)+\mathrm{I}_{T}(t) \mathrm{P}_{\mathrm{k}}^{S}(t)+t \mathrm{P}_{\mathrm{k}}^{S}(t) \mathrm{P}_{\mathrm{k}}^{T}(t)}{1-\left(\mathrm{P}_{\mathrm{k}}^{S}(t)-1\right)\left(\mathrm{P}_{\mathrm{k}}^{T}(t)-1\right)}
$$

in case $T$ is singular. And in case $T$ is regular, combine it with (3.1.3) to obtain

$$
\mathrm{I}_{R}(t)=\frac{\mathrm{I}_{S}(t)(1+t)^{n}-t^{n+1} \mathrm{P}_{\mathrm{k}}^{S}(t)+t(1+t)^{n} \mathrm{P}_{\mathrm{k}}^{S}(t)}{1-\left(\mathrm{P}_{\mathrm{k}}^{S}(t)-1\right)\left(\mathrm{P}_{\mathrm{k}}^{T}(t)-1\right)},
$$

where $n \geqslant 1$ is the dimension of $T$. As $S$ is not a hypersurface, its Poincaré series has increasing coefficients, so the power series $(1-t)\left(\mathrm{P}_{\mathrm{k}}^{S}(t)-1\right)$ has positive coefficients, and hence so has $(1-t)\left(\mathrm{P}_{\mathrm{k}}^{S}(t)-1\right)\left(\mathrm{P}_{\mathrm{k}}^{T}(t)-1\right)$. That is, the series $\left(\mathrm{P}_{\mathrm{k}}^{S}(t)-1\right)\left(\mathrm{P}_{\mathrm{k}}^{T}(t)-1\right)$ of order 2 has increasing coefficients. Moreover, the degree 2 coefficient $(\operatorname{edim} S)(\operatorname{edim} T)$ is greater than 1 as $S$ is not a hypersurface. From Lemma (3.5) (a) it now follows that the series $1 /\left[1-\left(\mathrm{P}_{\mathrm{k}}^{S}(t)-1\right)\left(\mathrm{P}_{\mathrm{k}}^{T}(t)-1\right)\right]$ has exponential growth. In (3.6.1) as well as in (3.6.2) the numerator has non-negative coefficients, so in either case the sequence $\left\{\mu^{i}(R)\right\}_{i \geqslant d}$ has exponential growth.

If $T$ is singular, then the series $(1-t) \mathrm{P}_{\mathrm{k}}^{T}(t)$ has non-negative coefficients, and as above $(1-t) \mathrm{P}_{\mathrm{k}}^{S}(t)$ has positive coefficients. Thus, the numerator in the expression

$$
(1-t) \mathrm{I}_{R}(t)=\frac{(1-t)\left(\mathrm{I}_{S}(t) \mathrm{P}_{\mathrm{k}}^{T}(t)+\mathrm{I}_{T}(t) \mathrm{P}_{\mathrm{k}}^{S}(t)+t \mathrm{P}_{\mathrm{k}}^{S}(t) \mathrm{P}_{\mathrm{k}}^{T}(t)\right)}{1-\left(\mathrm{P}_{\mathrm{k}}^{S}(t)-1\right)\left(\mathrm{P}_{\mathrm{k}}^{T}(t)-1\right)},
$$

which is derived from (3.6.1), is a power series of order $d \leqslant 1$ with positive coefficients. As the power series $1 /\left[1-\left(\mathrm{P}_{\mathrm{k}}^{S}(t)-1\right)\left(\mathrm{P}_{\mathrm{k}}^{T}(t)-1\right)\right]$ has non-negative coefficients and order 0 , it follows that $(1-t) \mathrm{I}_{R}(t)$ has positive coefficients in all degrees $i \geqslant d$. That is, the sequence $\left\{\mu^{i}(R)\right\}_{i \geqslant d}$ is increasing.

Finally, assume that $T$ is regular of dimension $n$. The Poincaré series $(1+t)^{n}$ and $\mathrm{P}_{\mathrm{k}}^{S}(t)$ satisfy the condition on $W(t)$ in Lemma (3.5) (b); see also Remark (3.6). By (3.6.2) the series $(1-t) \mathrm{I}_{R}(t)$ of order $d \leqslant 1$ can be expressed as a sum

$$
\begin{aligned}
(1-t) \mathrm{I}_{R}(t) & =(1-t) \frac{(1+t)^{n}}{1-\left(\mathrm{P}_{\mathrm{k}}^{S}(t)-1\right)\left(\mathrm{P}_{\mathrm{k}}^{T}(t)-1\right)} \mathrm{I}_{S}(t) \\
& +(1-t) \frac{\mathrm{P}_{\mathrm{k}}^{S}(t)}{1-\left(\mathrm{P}_{\mathrm{k}}^{S}(t)-1\right)\left(\mathrm{P}_{\mathrm{k}}^{T}(t)-1\right)}\left(t(1+t)^{n}-t^{n+1}\right) .
\end{aligned}
$$

The first summand has order depth $S \geqslant d$, and by Lemma (3.5)(b) it has nonnegative coefficients. Similarly, the second summand has order 1 and positive coefficients, also in degree 2 as $\beta_{1}^{S}(\mathrm{k})-\beta_{0}^{S}(\mathrm{k})=\operatorname{edim} S-1>0$. It follows that the 
power series $(1-t) \mathrm{I}_{R}(t)$ has positive coefficients in all degrees $i \geqslant d$, therefore, the sequence $\left\{\mu^{i}(R)\right\}_{i \geqslant d}$ is increasing.

(3.7) Remark. Let $R$ be artinian, and assume that $\mathrm{k}$ splits out of the maximal ideal $\mathfrak{m}$ - that is, Soc $R$ contains a minimal generator of $\mathfrak{m}$. If $R$ is not a hypersurface, i.e. edim $R$ is at least 2 , then it is a non-trivial fiber product of artinian local rings. Indeed, let $x, y_{1}, \ldots, y_{m}$ be a minimal set of generators of $\mathfrak{m}$, such that $x$ is in Soc $R$, then there is an isomorphism $R \cong R /(x) \times_{\mathrm{k}} R /\left(y_{1}, \ldots, y_{m}\right)$. By Theorem (3.4) the sequence $\left\{\mu^{i}(R)\right\}_{i \geqslant 0}$ is increasing and has exponential growth.

One can, however, do better for this particular kind of fiber products. Let $R$ be as in (3.7). If $\mathfrak{m}^{2}=0$ the sequence $\left\{\mu^{i}(R)\right\}_{i \geqslant 0}$ has even termwise exponential growth; see Example (1.5). It the next section - see Proposition (4.7) - the same conclusion is reached in the case $\mathfrak{m}^{2} \neq 0$, and this establishes part (3) of the Main Theorem.

\section{Artinian Rings}

In this section $(R, \mathfrak{m}, \mathrm{k})$ is artinian; the injective envelope of $\mathrm{k}$ is denoted $\mathrm{E}_{R}(\mathrm{k})$. The results in this section prepare the grounds for the proof of part (4) of the Main Theorem from the Introduction; it is given in the next section. The last result of this section establishes the Main Theorem's part (3).

(4.1) Let $M$ be a finitely generated $R$-module. We write $\ell_{R}(M)$ for the length of $M$ and Soc $M$ for its socle. It is straightforward to verify the following:

$\mathrm{k}$ is a direct summand of $M$ if and only if Soc $M \nsubseteq \mathfrak{m} M$.

For $i \geqslant 1$ we denote the $i^{\text {th }}$ syzygy in a minimal free resolution of $M$ by $M_{i}$; we set $M_{0}=M$. Since the differentials in a minimal free resolution are given by matrices with entries in $\mathfrak{m}$, there are equalities

$$
\operatorname{Soc} M_{i+1}=\operatorname{Soc} R^{\beta_{i}^{R}(M)} \quad \text { for all } i \geqslant 1 .
$$

(4.2) Recall the following special case of (1.0.1):

$$
\mu^{i}(R)=\beta_{i}^{R}\left(\mathrm{E}_{R}(\mathrm{k})\right) \quad \text { for all } i \geqslant 0 .
$$

If $R$ is not Gorenstein, then the equality $\ell_{R}\left(\mathrm{E}_{R}(\mathrm{k})\right)=\ell(R)$ and Lemma (A.1) yield

$$
\mu^{1}(R) \geqslant \mu^{0}(R) .
$$

Recall also that one has $\operatorname{rank}_{\mathrm{k}} \operatorname{Soc} R=\mu^{0}(R)$, and denote this number by $r$.

The next result contains a special case of Theorem (5.1), namely the one where the socle rank $r$ exceeds the embedding dimension.

(4.3) Proposition. Set $h=\max \left\{i \mid \mathfrak{m}^{i} \neq 0\right\}$ and assume that $R$ satisfies $h \geqslant 2$. If $r>\ell_{R}\left(R / \mathfrak{m}^{h}\right)-1$, then the sequence of Bass numbers $\left\{\mu^{i}(R)\right\}_{i \geqslant 0}$ is increasing and has termwise exponential growth of rate at least

$$
\frac{r}{\ell_{R}\left(R / \mathfrak{m}^{h}\right)-1} .
$$

Proof. The assumptions on $R$ force an inequality $r>2$; in particular $R$ is not Gorenstein. Indeed, let $e$ be the embedding dimension of $R$, then one has $r>e$, as $h \geqslant 2$ by assumption. If $e$ were 1 , then $R$ would be a hypersurface, so also $r$ would be 1 , which is impossible. Hence, one has $e \geqslant 2$ and $r>2$. 
By Proposition (A.2) we need only prove the inequality $\mu^{1}(R)>\mu^{0}(R)$, and to this end it suffices, by (4.2.2), to show $\mu^{1}(R) \neq r$. Assume, towards a contradiction, that one has $\mu^{1}(R)=r$. Set $E=\mathrm{E}_{R}(\mathrm{k})$ and consider the exact sequence

$$
0 \rightarrow E_{2} \rightarrow R^{r} \rightarrow R^{r} \rightarrow E \rightarrow 0
$$

that comes from the minimal free resolution of $E$. By (4.1.2) and additivity of length, the sequence yields (in)equalities

$$
r^{2}=\operatorname{rank}_{\mathrm{k}} \operatorname{Soc} R^{r}=\operatorname{rank}_{\mathrm{k}} \operatorname{Soc} E_{2} \leqslant \ell_{R}\left(E_{2}\right)=\ell_{R}(E) .
$$

By additivity of length, the assumptions on $R$, and the containment $\mathfrak{m}^{h} \subseteq$ Soc $R$ there are (in)equalities

$$
\ell(R)=\ell_{R}\left(R / \mathfrak{m}^{h}\right)+\ell_{R}\left(\mathfrak{m}^{h}\right)<1+r+\operatorname{rank}_{\mathbf{k}} \mathfrak{m}^{h} \leqslant 1+2 r .
$$

By the equality $\ell_{R}(E)=\ell(R)$, the last two displays combine to yield $r^{2} \leqslant 1+2 r$, which implies $r \leqslant 2$, a contradiction.

(4.4) Lemma. If $\mathrm{k}$ is a direct summand of the first syzygy of $\mathrm{E}_{R}(\mathrm{k})$, then one has $\mu^{1}(R)>\mu^{0}(R)$.

Proof. By (4.2.2) it is enough to rule out the possibility of an equality $\mu^{1}(R)=r$. Suppose this equality holds. For brevity, set $E=\mathrm{E}_{R}(\mathrm{k})$. By hypothesis, there is an isomorphism $E_{1} \cong \mathrm{k} \oplus N$ for some finitely generated $R$-module $N$ and, therefore, there is an isomorphism of syzygies $E_{2} \cong \mathfrak{m} \oplus N_{1}$. From the exact sequence $0 \rightarrow E_{2} \rightarrow R^{r} \rightarrow R^{r} \rightarrow E \rightarrow 0$ one obtains $\ell_{R}(\mathfrak{m})+\ell_{R}\left(N_{1}\right)=\ell_{R}(E)$ by additivity of length. Since $\ell_{R}(E)=\ell_{R}(R)=\ell_{R}(\mathfrak{m})+1$, it follows that $N_{1}$ is a k-vector space of rank 1 . Hence there is an exact sequence $0 \rightarrow \mathrm{k} \rightarrow R^{r-1} \rightarrow N \rightarrow 0$, where the surjective homomorphism is given by a matrix with entries in $\mathfrak{m}$, as $N$ is a submodule of $\mathfrak{m} R^{r}$, cf. 4.1.2). Thus, we have Soc $R^{r-1}=\mathrm{k}$, and the ensuing equality of ranks $r(r-1)=1$ is absurd.

(4.5) Lemma. Assume that $R$ is not Gorenstein, and set $E=\mathrm{E}_{R}(\mathrm{k})$. Let $n$ be an integer such that Soc $E_{1}$ is contained in $\mathfrak{m}^{n} E_{1}$. Then one has $n<\max \left\{i \mid \mathfrak{m}^{i} \neq 0\right\}$ and $\operatorname{Soc} R \subseteq \mathfrak{m}^{n+1}$.

Proof. Set $h=\max \left\{i \mid \mathfrak{m}^{i} \neq 0\right\}$, then $\mathfrak{m}^{h} E_{1}=0$ as $E_{1}$ is a submodule of $\mathfrak{m} R^{r}$; and the inequality $n<h$ follows.

Let $e_{1}, \ldots, e_{r}$ be the standard basis for $R^{r}$. Choose a minimal set $\varepsilon_{1}, \ldots, \varepsilon_{r}$ of generators of $E$ and consider the short exact sequence $0 \rightarrow E_{1} \rightarrow R^{r} \rightarrow E \rightarrow 0$, where the surjection maps $e_{i}$ to $\varepsilon_{i}$. Suppose that Soc $R$ is not contained in $\mathfrak{m}^{n+1}$ and choose an element $x$ in $(\operatorname{Soc} R) \backslash \mathfrak{m}^{n+1}$. Since $E$ is a faithful $R$-module, there is an index $i$ such that the element $x \varepsilon_{i}$ is non-zero; it clearly belongs to Soc $E$. Similarly, by the definition of $h$, it follows that $\mathfrak{m}^{h} E$ is a non-zero submodule of Soc $E$. Since $\operatorname{rank}_{\mathbf{k}} \operatorname{Soc} E=1$, there exists an element $y$ in $\mathfrak{m}^{h}$ and an index $j$ such that $y \varepsilon_{j}=x \varepsilon_{i}$. The element $z=x e_{i}-y e_{j}$ is in the socle of $E_{1}$, as $x$ and $y$ are in the socle of $R$. As $y$ is in $\mathfrak{m}^{n+1}$ but $x$ is not, the element $z$ is not in $\mathfrak{m}^{n} E_{1} \subseteq \mathfrak{m}^{n+1} R^{r}$, which contradicts the assumption that $\operatorname{Soc} E_{1}$ is contained in $\mathfrak{m}^{n} E_{1}$.

(4.6) Remark. Set $E=\mathrm{E}_{R}(\mathrm{k})$. If $\mathrm{k}$ is a direct summand of $\mathfrak{m}$, that is $\operatorname{Soc} R \nsubseteq \mathfrak{m}^{2}$, then it follows from Lemma (4.5) that Soc $E_{1} \nsubseteq \mathfrak{m} E_{1}$. Thus, $\mathrm{k}$ is a direct summand of $E_{1}$, cf. (4.1.1), and then Lemma (4.4) yields the inequality $\mu^{1}(R)>\mu^{0}(R)$.

Finally, Proposition (A.3) together with Remark (4.6) gives us: 
(4.7) Proposition. Let $R$ be an artinian local ring of embedding dimension at least 2. If Soc $R \nsubseteq \mathfrak{m}^{2}$, then the sequence of Bass numbers $\left\{\mu^{i}(R)\right\}_{i \geqslant 0}$ is increasing and it has termwise exponential growth.

\section{RINGS WITH $\mathfrak{m}^{3}=0$}

The theorem below is part (4) of the Main Theorem from the Introduction.

(5.1) Theorem. Let $(R, \mathfrak{m})$ be local with $\mathfrak{m}^{3}=0$. If $R$ is not Gorenstein, then the Bass sequence $\left\{\mu^{i}(R)\right\}_{i \geqslant 0}$ is increasing and has termwise exponential growth.

Proof. Fix the following notation

$$
a=\operatorname{rank}_{\mathrm{k}} \mathfrak{m}^{2}, \quad e=\operatorname{rank}_{\mathrm{k}} \mathfrak{m} / \mathfrak{m}^{2}, \quad \text { and } \quad r=\operatorname{rank}_{\mathrm{k}} \operatorname{Soc} R .
$$

The embedding dimension $e$ is at least 2 , as $R$ is not a hypersurface, and for $e=2$ the statement is contained in Corollary (2.7), cf. Remark (2.8). In the rest of the proof, we assume $e \geqslant 3$. In view of Example (1.5) we can assume that $\mathfrak{m}^{2}$ is not 0 , i.e. $a>0$. Set $E=\mathrm{E}_{R}(\mathrm{k})$. For $i \geqslant 0$ set $\mu^{i}=\mu^{i}(R)$ and recall from (4.2.1) that $\mu^{i}=\beta_{i}^{R}(E)$. The containment $\mathfrak{m}^{2} \subseteq \operatorname{Soc} R$ implies an inequality $a \leqslant r$.

If $a<r$, then Soc $R$ is not contained in $\mathfrak{m}^{2}$ and Remark (4.6) gives the inequality $\mu^{1}>\mu^{0}$. Then Theorem (A.5) (a) applies to the module $E$, so the sequence $\left\{\mu^{i}\right\}_{i \geqslant 0}$ is increasing and it has termwise exponential growth.

In the remainder of the proof assume $a=r$. From the equalities $\ell_{R}(E)=\ell(R)$ and $1=\operatorname{rank}_{\mathrm{k}} \operatorname{Soc} E=\operatorname{rank}_{\mathrm{k}} \mathfrak{m}^{2} E$ one gets $\operatorname{rank}_{\mathrm{k}} \mathfrak{m} E / \mathfrak{m}^{2} E=\ell(R)-r-1=e$. By [19, lem. 3.3] — see also (A.4.2 — there is an inequality

$$
\mu^{1} \geqslant e \mu^{0}-\operatorname{rank}_{\mathbf{k}} \mathfrak{m} E / \mathfrak{m}^{2} E=e(r-1),
$$

and equality holds if and only if $\mathrm{k}$ is not a direct summand of $E_{1}$. Since $e \geqslant 3$ and $r \geqslant 2$, an equality $\mu^{1}=\mu^{0}$ would imply $r \geqslant e(r-1) \geqslant r+2 r-3 \geqslant r+1$, which is absurd. Thus, the inequality $\mu^{1}>\mu^{0}$ holds, cf. (4.2.2). Now Theorem (A.5) (a) applies to the module $E$, so the sequence $\left\{\mu^{i}\right\}_{i \geqslant 0}$ is increasing with termwise exponential growth except, possibly, when $a=e=r$.

Assume now that all three invariants $a, e$, and $r$ are equal. Note that (11) yields

$$
\mu^{1} \geqslant(r-1) \mu^{0} .
$$

By [19, lem. 3.3] - see also (A.4.3 — there are inequalities

$$
\mu^{i+1} \geqslant r\left(\mu^{i}-\mu^{i-1}\right) \text { for } i \geqslant 1
$$

and equality holds if $i \geqslant 2$ and $\mathrm{k}$ is not a summand of either syzygy $E_{i}$ and $E_{i-1}$.

Consider the case where the common value of $a, e$, and $r$ is at least 4 . The quantity $A=\frac{1}{2}\left(r+\sqrt{r^{2}-4 r}\right)$ is then a real number greater than or equal to 2 . We claim that the inequality $\mu^{i+1} \geqslant A \mu^{i}$ holds for all $i \geqslant 0$. The proof is by induction on $i$. The base case $i=0$ is furnished by (2) and the induction step follows from (3):

$$
\mu^{i+1} \geqslant r\left(\mu^{i}-\mu^{i-1}\right) \geqslant r\left(\mu^{i}-A^{-1} \mu^{i}\right)=r\left(1-A^{-1}\right) \mu^{i}=A \mu^{i},
$$

where the last equality follows as $A$ is a solution to the equation $r\left(1-x^{-1}\right)=x$.

As $e \geqslant 3$ we are left with only one case to consider, namely $a=e=r=3$. First we prove that the sequence $\left\{\mu^{i}\right\}_{i \geqslant 0}$ is increasing and that $\mathrm{k}$ is a direct summand of one of the first four syzygies of $E$. If $\mathrm{k}$ is a direct summand of $E_{1}$ or $E_{2}$, then the 
sequence $\left\{\mu^{i}\right\}_{i \geqslant 0}$ is increasing by Theorem (A.5). Assume now that $\mathrm{k}$ is not a direct summand of $E_{1}$, then (11) yields

$$
\mu^{1}=e(r-1)=6 .
$$

Assume also that $\mathrm{k}$ is not a direct summand of $E_{2}$. In the computation

$$
\operatorname{rank}_{\mathrm{k}} \mathfrak{m} E_{2}=\operatorname{rank}_{\mathrm{k}} \operatorname{Soc} E_{2}=\operatorname{rank}_{\mathrm{k}} \operatorname{Soc} R^{6}=18
$$

the first equality follows from (4.1.1) and the second from (4.1.2). Combined with length computations in the minimal free resolution of $E$, this gives

$$
\mu^{2}=\ell_{R}\left(E_{2}\right)-\ell_{R}\left(\mathfrak{m} E_{2}\right)=4 \ell_{R}(R)-18=10 .
$$

In particular, one has $\mu^{2}>\mu^{1}$. If $\mathrm{k}$ is a direct summand of $E_{3}$, then the sequence $\left\{\mu^{i}\right\}_{i \geqslant 0}$ is increasing by (A.5) (b). If $\mathrm{k}$ is not a direct summand of $E_{3}$, then (3) yields

$$
\mu^{3}=3\left(\mu^{2}-\mu^{1}\right)=12 .
$$

If $\mathrm{k}$ were not a direct summand of $E_{4}$, then (3) would yield $\mu^{4}=3\left(\mu^{3}-\mu^{2}\right)=6<\mu^{3}$ which is impossible by Theorem (A.5) (b). Thus, $\mathrm{k}$ is a direct summand of $E_{4}$, and the sequence $\left\{\mu^{i}\right\}_{i \geqslant 0}$ is increasing by (A.5) (b) as one has $\mu^{3}>\mu^{2}$.

Finally, we can conclude that the growth of the series $\left\{\mu^{i}\right\}_{i \geqslant 0}$ is termwise exponential. Since $\mathrm{k}$ is a direct summand of a syzygy of $E$, the radius of convergence of the power series $\mathrm{P}_{E}^{R}(t)=\mathrm{I}_{R}(t)$ is bounded above by that of $\mathrm{P}_{\mathrm{k}}^{R}(t)$. The opposite inequality always holds by [19, prop. 1.1], so the two power series have the same radius of convergence $\rho$. As $R$ is not complete intersection, the sequence $\left\{\beta_{i}^{R}(\mathbf{k})\right\}_{i \geqslant 0}$ has exponential growth, so one has $\rho<1$. From work of Sun [25, thm. 1.2] it now follows that the sequence $\left\{\mu^{i}\right\}_{i \geqslant 0}$ has termwise exponential growth.

\begin{tabular}{|c|c|c|c|c|c|c|c|}
\hline \multirow[t]{3}{*}{$a$} & \multirow{3}{*}{$a=0$} & \multirow{3}{*}{$a<r$} & \multicolumn{5}{|c|}{$a=r$} \\
\hline & & & \multirow[t]{2}{*}{$e<r$} & \multicolumn{2}{|c|}{$e=r$} & \multirow[t]{2}{*}{$e=r+1$} & \multirow[t]{2}{*}{$e \geqslant r+2$} \\
\hline & & & & $2 \leqslant r \leqslant 3$ & $4 \leqslant r$ & & \\
\hline$A$ & $e=r$ & $r-a+\frac{r-a}{a+e}$ & $\frac{r}{e}$ & 2 & $\frac{r+\sqrt{r^{2}-4 r}}{2}$ & $\frac{r^{2}-1}{r}$ & $e-r$ \\
\hline
\end{tabular}

(5.2) Scholium. Let $R$ be as in Theorem (5.1). The table below gives a lower bound for the rate $A$ of the termwise exponential growth of the Bass sequence $\left\{\mu^{i}(R)\right\}_{i \geqslant 0}$ in terms of the invariants $a=\operatorname{rank}_{\mathfrak{k}} \mathfrak{m}^{2}, e=\operatorname{rank}_{\mathfrak{k}} \mathfrak{m} / \mathfrak{m}^{2}$, and $r=\operatorname{rank}_{\mathrm{k}} \operatorname{Soc} R$.

For rings with $a=e=r=3$, Backelin and Fröberg [6] give closed form expressions for the possible Poincaré series $\mathrm{P}_{\mathrm{k}}^{R}(t)$, and one can verify directly that the radius of convergence is less than $\frac{1}{2}$. Sun's [25, thm. 1.2] then yields the lower bound 2 for $A$. In all other cases, the bound follows by inspection of Example (1.4), the proof of Theorem (A.5) (a), the proof of Theorem (5.1), and Peeva's proof of [21, prop. 3].

\section{TETER RINGS}

Following Huneke and Vraciu [14], we say that $R$ is Teter if there exists an artinian Gorenstein local ring $Q$ such that $R \cong Q / \operatorname{Soc} Q$.

(6.1) Let $Q$ be an artinian Gorenstein local ring which is not a field. Denote by k its residue field. Set $R=Q / \operatorname{Soc} Q$. The maximal ideal of $Q$ is isomorphic to 
$\operatorname{Hom}_{Q}(R, Q)$, which is the injective hull of $\mathrm{k}$ as an $R$-module. Thus, $R$ is Gorenstein if and only if $\operatorname{edim} Q=1$, in which case both $Q$ and $R$ are hypersurfaces.

If $Q$ is not a hypersurface, i.e. edim $Q \geqslant 2$, then one has $\operatorname{edim} R=\operatorname{edim} Q$ and works of Avramov and Levin [20, thm. 2.9] and Herzog and Steurich [13, prop. 1] provide the following expression for the Bass series of $R$ :

$$
\mathrm{I}_{R}(t)=\frac{\mathrm{P}_{\mathrm{k}}^{Q}(t)-1}{t\left(1-t^{2} \mathrm{P}_{\mathrm{k}}^{Q}(t)\right)}
$$

Notice the equality $\mu^{0}(R)=\operatorname{edim} R$.

(6.2) For each positive integer $e$ set

$$
\begin{aligned}
& \Upsilon_{i}^{e}=\left(\begin{array}{c}
e \\
i
\end{array}\right)^{-1}\left(\sum_{j=0}^{i+1}\left(\begin{array}{c}
e-1 \\
j
\end{array}\right)\right) \quad \text { for } i \in\{0, \ldots, e\} \text { and } \\
& \Upsilon^{e}=\min \left\{\Upsilon_{i}^{e} \mid 0 \leqslant i \leqslant e\right\} .
\end{aligned}
$$

The quantities $\Upsilon^{e}$ are used below to provide lower bounds for the growth rate of Bass numbers, and we make a few observations on how to compute or estimate their values. Notice that one has $\Upsilon_{0}^{e}=e$ and $\Upsilon_{e}^{e}=2^{e-1}$ for every $e \geqslant 1$. Moreover, one has $\Upsilon_{1}^{2}=1$, and for $e \geqslant 3$ it is straightforward to establish the inequalities

$$
\begin{aligned}
& \Upsilon_{1}^{e} \leqslant \frac{e}{2} \\
& 1<1+\left(\begin{array}{c}
e \\
i
\end{array}\right)^{-1}\left(\begin{array}{c}
e-1 \\
i+1
\end{array}\right) \leqslant \Upsilon_{i}^{e} \quad \text { for } \quad 1 \leqslant i \leqslant\left\lfloor\frac{e}{2}\right\rfloor \quad \text { and } \\
& \Upsilon_{i}^{e} \leqslant \Upsilon_{i+1}^{e} \text { for }\left\lfloor\frac{e}{2}\right\rfloor \leqslant i \leqslant e-1 .
\end{aligned}
$$

In particular, one has

$$
\Upsilon^{1}=1=\Upsilon^{2} \quad \text { and } \quad 1<\Upsilon^{e}=\min \left\{\Upsilon_{i}^{e} \mid 1 \leqslant i \leqslant\left\lfloor\frac{e}{2}\right\rfloor\right\} \text { for } e \geqslant 3 .
$$

Direct computations yield $\Upsilon^{e}=\Upsilon_{\left\lfloor\frac{e}{2}\right\rfloor}^{e}$ for $e \leqslant 9$ but $\Upsilon^{10}=\Upsilon_{4}^{10}$. To find $\Upsilon^{e}$ for larger values of $e$ it is useful to know that $\Upsilon_{i}^{e}$ is an upwards convex function of $i$; Roger W. Barnard proved this upon request. Thus, if $n$ is an integer between 1 and $\left\lfloor\frac{e}{2}\right\rfloor$ and there are inequalities $\Upsilon_{n-1}^{e} \geqslant \Upsilon_{n}^{e} \leqslant \Upsilon_{n+1}^{e}$, then one has $\Upsilon^{e}=\Upsilon_{n}^{e}$.

(6.3) Lemma. Let $R$ be artinian of embedding dimension $e \geqslant 1$. For every real number $A<\Upsilon^{e}$ the formal power series $\left(1-A t+A t^{3}\right) \mathrm{P}_{\mathrm{k}}^{R}(t)$ has positive coefficients, and for $A=\Upsilon^{e}$ it has non-negative coefficients.

Proof. By [2, 7.1] there exist non-negative integers $\varepsilon_{i}$ such that

$$
\mathrm{P}_{\mathrm{k}}^{R}(t)=\frac{\prod_{i=1}^{i=\infty}\left(1+t^{2 i-1}\right)^{\varepsilon_{2 i-1}}}{\prod_{i=1}^{i=\infty}\left(1-t^{2 i}\right)^{\varepsilon_{2 i}}}=\frac{(1+t)^{\varepsilon_{1}}}{1-t^{2}} \cdot \frac{\prod_{i=2}^{i=\infty}\left(1+t^{2 i-1}\right)^{\varepsilon_{2 i-1}}}{\left(1-t^{2}\right)^{\varepsilon_{2}-1} \prod_{i=2}^{i=\infty}\left(1-t^{2 i}\right)^{\varepsilon_{2 i}}},
$$

and one has $\varepsilon_{2}-1 \geqslant 0$ as $R$ is not regular; see [2, thm. 7.3.2]. By [2, cor. 7.1.5] there is an equality $\varepsilon_{1}=e$, so the factor $F=\frac{(1+t)^{\varepsilon_{1}}}{1-t^{2}}$ can be rewritten as

$$
F=\frac{(1+t)^{e-1}}{1-t}=(1+t)^{e-1} \sum_{i=0}^{\infty} t^{i}=\sum_{i=0}^{e-2}\left(\sum_{j=0}^{i}\left(\begin{array}{c}
e-1 \\
j
\end{array}\right)\right) t^{i}+\sum_{i=e-1}^{\infty} 2^{e-1} t^{i} .
$$

The power series $\left(1-A t+A t^{3}\right) \mathrm{P}_{\mathrm{k}}^{R}(t)$ has positive/non-negative coefficients if the series $\left(1-A t+A t^{3}\right) F$ has positive/non-negative coefficients. From the computation 


$$
\begin{aligned}
\left(1-A t+A t^{3}\right) F & =\sum_{i=0}^{e+1}\left(\sum_{j=0}^{i}\left(\begin{array}{c}
e-1 \\
j
\end{array}\right)-A\left(\left(\begin{array}{c}
e-1 \\
i-2
\end{array}\right)+\left(\begin{array}{c}
e-1 \\
i-1
\end{array}\right)\right)\right) t^{i}+\sum_{i=e+2}^{\infty} 2^{e-1} t^{i} \\
& =1+\sum_{i=1}^{e+1}\left(\sum_{j=0}^{i}\left(\begin{array}{c}
e-1 \\
j
\end{array}\right)-A\left(\begin{array}{c}
e \\
i-1
\end{array}\right)\right) t^{i}+\sum_{i=e+2}^{\infty} 2^{e-1} t^{i} \\
& =1+\sum_{i=0}^{e}\left(\sum_{j=0}^{i+1}\left(\begin{array}{c}
e-1 \\
j
\end{array}\right)-A\left(\begin{array}{c}
e \\
i
\end{array}\right)\right) t^{i+1}+\sum_{i=e+2}^{\infty} 2^{e-1} t^{i}
\end{aligned}
$$

it follows that $\left(1-A t+A t^{3}\right) F$ has non-negative coefficients if and only if $A \leqslant \Upsilon^{e}$ and positive coefficients if $A<\Upsilon^{e}$.

The next result establishes part (5) of the Main Theorem.

(6.4) Theorem. Let $R$ be Teter of embedding dimension $e$ at least 2.

(a) If $e=2$, then $\mu^{0}(R)=2$ and $\mu^{i}(R)=3\left(2^{i-1}\right)$ for $i \geqslant 1$.

(b) If $e>2$, then $\Upsilon^{e}>1$ and the formal power series $\left(1-\Upsilon^{e} t\right) \mathrm{I}_{R}(t)$ has nonnegative coefficients.

In particular, the sequence of Bass numbers $\left\{\mu^{i}(R)\right\}_{i \geqslant 0}$ is increasing and has termwise exponential growth.

Proof. If $e=2$, then $R$ is Golod; see [2, prop. 5.3.4]. Since $R$ is Teter, it follows from (6.1.1) that $\mu^{0}(R)=e=2$. Thus, part (a) follows from Remark (2.10).

Assume now that $e>2$, then $\Upsilon^{e}>1$ by (6.2.1). To prove part (b), it is sufficient to show that the series $\left(1-\Upsilon^{e} t\right)\left(1+t \mathrm{I}_{R}(t)\right)$ has non-negative coefficients. To this end, let $Q$ be an artinian Gorenstein ring such that $R \cong Q / \operatorname{Soc} Q$. From (6.1.1) one obtains

$$
1+t \mathrm{I}_{R}(t)=\left(1+t^{3} \mathrm{I}_{R}(t)\right) \mathrm{P}_{\mathrm{k}}^{Q}(t)=\left(1-t^{2}+t^{2}\left(1+t \mathrm{I}_{R}(t)\right)\right) \mathrm{P}_{\mathrm{k}}^{Q}(t) .
$$

Set $1+t \mathrm{I}_{R}(t)=\sum_{i=0}^{\infty} a_{i} t^{i}$ and $\mathrm{P}_{\mathrm{k}}^{Q}(t)=\sum_{i=0}^{\infty} b_{i} t^{i}$. Now (1) yields relations

$$
\begin{aligned}
& a_{0}=b_{0}=1, \\
& a_{1}=b_{1}, \\
& a_{2}=b_{2}-b_{0}+b_{0} a_{0}=b_{2},
\end{aligned}
$$

and for $n \geqslant 2$ :

$$
a_{n}=b_{n}-b_{n-2}+\sum_{i=0}^{n-2} b_{i} a_{n-2-i} .
$$

The coefficients of the series $\left(1-\Upsilon^{e} t\right)\left(1+t \mathrm{I}_{R}(t)\right)$ may now be expressed as

$$
\begin{aligned}
& a_{1}-\Upsilon^{e} a_{0}=b_{1}-\Upsilon^{e} b_{0}, \\
& a_{2}-\Upsilon^{e} a_{1}=b_{2}-\Upsilon^{e} b_{1}, \\
& a_{3}-\Upsilon^{e} a_{2}=b_{3}-\Upsilon^{e} b_{2}+\Upsilon^{e} b_{0}+b_{0}\left(a_{1}-\Upsilon^{e} a_{0}\right),
\end{aligned}
$$

and for $n \geqslant 3$ :

$$
a_{n}-\Upsilon^{e} a_{n-1}=b_{n}-\Upsilon^{e} b_{n-1}+\Upsilon^{e} b_{n-3}+\sum_{i=0}^{n-3} b_{i}\left(a_{n-2-i}-\Upsilon^{e} a_{n-3-i}\right) .
$$


Since $R$ is not a hypersurface, the $\operatorname{ring} Q$ is not a hypersurface and $\operatorname{edim} Q=e$. By Lemma (6.3) the series $\left(1-\Upsilon^{e} t+\Upsilon^{e} t^{3}\right) \mathrm{P}_{\mathrm{k}}^{Q}(t)$ has non-negative coefficients, which means that $b_{n}-\Upsilon^{e} b_{n-1}+\Upsilon^{e} b_{n-3} \geqslant 0$ for all $n \geqslant 1$. It now follows by recursion that $a_{n}-\Upsilon^{e} a_{n-1} \geqslant 0$ for all $n \geqslant 1$.

\section{Appendix. Free RESOlutions OVER ARTINIAN Rings}

Here we collect a few results on the growth of Betti numbers of modules over an artinian local ring $(R, \mathfrak{m}, \mathrm{k})$. Their main application in this paper is to the module $\mathrm{E}_{R}(\mathrm{k})$, the injective hull of $\mathrm{k}$.

The first result, Lemma (A.1) below, proves the characterization (1.6) of Gorenstein rings. Indeed, let $Q$ be a Cohen-Macaulay local ring of dimension $d$ and let $\boldsymbol{x}=x_{1}, \ldots, x_{d}$ be a $Q$-regular sequence. Then $R=Q /(\boldsymbol{x})$ is artinian, and one has

$$
\mu^{d+i}(Q)=\mu^{i}(R)=\beta_{i}^{R}\left(\mathrm{E}_{R}(\mathrm{k})\right) \text { for all } i \geqslant 0 ;
$$

see (4.2.1) and [8, prop. 1.2.(c)]. If $Q$ is not Gorenstein, then $R$ is not Gorenstein and Lemma A.1 applies to $\mathrm{E}_{R}(\mathrm{k})$ and yields $\mu^{d+i}(Q) \geqslant 2$ for all $i \geqslant 0$.

(A.1) Lemma. Let $M$ be a non-free finitely generated $R$-module. If $\ell(R)$ divides $\ell_{R}(M)$, then one has

$$
\beta_{i}^{R}(M) \geqslant 2 \text { for all } i \geqslant 0 .
$$

Moreover, if $\ell_{R}(M)=\ell(R)$, then the inequality $\beta_{1}^{R}(M) \geqslant \beta_{0}^{R}(M)$ holds.

Proof. Since $M$ is not free, we have $\beta_{i}^{R}(M) \geqslant 1$ for all $i \geqslant 0$. For every $j \geqslant 0$, a length computation based on the exact sequence

$$
0 \rightarrow M_{j+1} \rightarrow R^{\beta_{j}^{R}(M)} \rightarrow \cdots \rightarrow R^{\beta_{0}^{R}(M)} \rightarrow M \rightarrow 0
$$

shows that $\ell_{R}(M) \equiv \pm \ell_{R}\left(M_{j+1}\right)(\bmod \ell(R))$. By assumption, $\ell(R)$ divides $\ell_{R}(M)$, so it follows that $\ell(R)$ divides $\ell_{R}\left(M_{j+1}\right)$; in particular, we have $\ell(R) \leqslant \ell_{R}\left(M_{j+1}\right)$.

Assume that $\beta_{j}^{R}(M)=1$ for some $j \geqslant 0$. Then the embedding $0 \rightarrow M_{j+1} \rightarrow R$ yields $\ell(R)>\ell_{R}\left(M_{j+1}\right)$, which contradicts the inequality obtained above. Therefore, $\beta_{i}^{R}(M)$ is at least 2 for all $i \geqslant 0$.

Now assume that $\ell_{R}(M)=\ell(R)$. For $j=1$ the sequence (11) gives

$$
\ell_{R}\left(M_{2}\right)=\left(\beta_{1}^{R}(M)-\beta_{0}^{R}(M)+1\right) \ell(R) .
$$

As $\ell_{R}\left(M_{2}\right)>0$, this forces the desired inequality.

In the following, $r$ denotes the socle rank of $R$. We set $h=\max \left\{i \mid \mathfrak{m}^{i} \neq 0\right\}$ and adopt the notation from (4.1). For a finitely generated $R$-module $M$, the rank of the largest k-vector space that is a direct summand of $M$ is called the k-rank of $M$.

The next proposition applies to rings of large socle rank, compared to the length of the ring. It complements a result of Gasharov on Peeva [10, prop. (2.2)] that applies to rings of large embedding dimension.

(A.2) Proposition. Assume that $h \geqslant 2$ and $r>\ell_{R}\left(R / \mathfrak{m}^{h}\right)-1$. For every finitely generated non-free $R$-module $M$, the sequence $\left\{\beta_{i}^{R}(M)\right\}_{i \geqslant 1}$ is increasing, and for $i \geqslant 2$ the next inequality holds

$$
\beta_{i+1}^{R}(M) \geqslant \frac{r}{\ell_{R}\left(R / \mathfrak{m}^{h}\right)-1} \beta_{i}^{R}(M) .
$$


Proof. Note that the assumption $h \geqslant 2$ yields $\ell_{R}\left(R / \mathfrak{m}^{h}\right) \geqslant 2$, thus the quotient $r /\left(\ell_{R}\left(R / \mathfrak{m}^{h}\right)-1\right)$ is well-defined. For $i \geqslant 1$ set $b_{i}=\beta_{i}^{R}(M)$ and let $s_{i}$ be the k-rank of $M_{i}$. Write the syzygy $M_{i+1}$ as a direct sum $M_{i+1} \cong \mathrm{k}^{s_{i+1}} \oplus N^{(i+1)}$, where $\mathrm{k}$ is not a direct summand of $N^{(i+1)}$. The isomorphism $\operatorname{Soc} R^{b_{i}} \cong \mathrm{k}^{s_{i+1}} \oplus \operatorname{Soc} N^{(i+1)}$, see (4.1.2), explains the second equality below.

$$
\begin{aligned}
r b_{i}=\operatorname{rank}_{\mathrm{k}} \operatorname{Soc} R^{b_{i}} & =s_{i+1}+\operatorname{rank}_{\mathrm{k}} \operatorname{Soc} N^{(i+1)} \\
& \leqslant s_{i+1}+\ell_{R}\left(\mathfrak{m} N^{(i+1)}\right) \\
& =s_{i+1}+\ell_{R}\left(N^{(i+1)}\right)-\beta_{0}^{R}\left(N^{(i+1)}\right) \\
& =s_{i+1}+\ell_{R}\left(N^{(i+1)}\right)-\left(b_{i+1}-s_{i+1}\right) \\
& \leqslant s_{i+1}+\left(b_{i+1}-s_{i+1}\right) \ell\left(R / \mathfrak{m}^{h}\right)-\left(b_{i+1}-s_{i+1}\right) \\
& =\left(\ell\left(R / \mathfrak{m}^{h}\right)-1\right) b_{i+1}+\left(2-\ell\left(R / \mathfrak{m}^{h}\right)\right) s_{i+1} \\
& \leqslant\left(\ell\left(R / \mathfrak{m}^{h}\right)-1\right) b_{i+1}
\end{aligned}
$$

The first inequality uses the containment $\operatorname{Soc} N^{(i+1)} \subseteq \mathfrak{m} N^{(i+1)}$, which holds as k is not a direct summand of $N^{(i+1)}$; see (4.1.1). The second inequality follows as $N^{(i+1)}$, being a summand of a syzygy, is an $R / \mathfrak{m}^{h}$ module generated by $b_{i+1}-s_{i+1}$ elements. The last inequality holds as we have $\ell_{R}\left(R / \mathfrak{m}^{h}\right) \geqslant 2$.

Applied to the module $\mathrm{E}_{R}(\mathrm{k})$, the next result establishes the termwise exponential growth of the sequence $\left\{\mu^{i}(R)\right\}_{i \geqslant 0}$ stated in Proposition (4.7), see (4.2.1). If there is more than one minimal generator of the maximal ideal in the socle of $R$, then Proposition (A.3) gives a higher rate of growth than Peeva's [21, lem. 6].

(A.3) Proposition. Assume that $R$ satisfies $\mathfrak{m}^{2} \neq 0$, Soc $R \nsubseteq \mathfrak{m}^{2}$ and edim $R \geqslant 2$. For every finitely generated non-free $R$-module $M$, the sequence $\left\{\beta_{i}^{R}(M)\right\}_{i \geqslant 1}$ is increasing, and for $i \geqslant 2$ the next inequality holds

$$
\beta_{i+1}^{R}(M) \geqslant\left(r-\operatorname{rank}_{\mathrm{k}}\left(\mathfrak{m}^{2} \cap \operatorname{Soc} R\right)\right)\left(1+\frac{1}{\ell(R)-1}\right) \beta_{i}^{R}(M) .
$$

Proof. For every $i \geqslant 0$ set $b_{i}=\beta_{i}^{R}(M)$. By assumption there is a minimal generator of $\mathfrak{m}$ in $\operatorname{Soc} R$. As $\operatorname{Soc} R^{b_{1}}$ is contained in $M_{2}$, this generator gives $b_{1}$ elements in $M_{2} \backslash \mathfrak{m} M_{2}$ that are linearly independent modulo $\mathfrak{m} M_{2}$. It follows from the assumptions on $R$ that $\operatorname{rank}_{\mathrm{k}} \operatorname{Soc} R$ is at least 2, so there are elements in $M_{2}$ which are not in the span of these $b_{1}$ elements. This proves the inequality $b_{2}>b_{1}$.

Set $A=r-\operatorname{rank}_{\mathrm{k}}\left(\mathfrak{m}^{2} \cap \operatorname{Soc} R\right)$. By assumption $A$ is at least 1 , and since $\mathfrak{m}^{2} \neq 0$ there is a minimal generator of $\mathfrak{m}$ outside the socle, so $e \geqslant A+1$. For $i \geqslant 2$ write $M_{i}$ as a direct sum $M_{i} \cong \mathrm{k}^{s_{i}} \oplus N^{(i)}$, where $\mathrm{k}$ is not a summand of $N^{(i)}$. By (4.1.2) we have Soc $M_{i}=\operatorname{Soc} R^{b_{i-1}}$. A k-vector space of rank $A b_{i-1}$ is a direct summand of $\mathfrak{m} R^{b_{i-1}}$ and hence in Soc $M_{i} \backslash \mathfrak{m} M_{i}$. In particular, there is an inequality

$$
s_{i} \geqslant A b_{i-1} \text {. }
$$

There is an isomorphism $M_{i+1} \cong \mathfrak{m}^{s_{i}} \oplus\left(N^{(i)}\right)_{1}$, so $M_{i+1}$ decomposes as a direct sum $\mathfrak{m}^{s_{i}} \oplus \mathrm{k}^{s} \oplus N$, where $\mathrm{k}$ is not a summand of $N$. Notice the equality $A s_{i}+s=s_{i+1}$; 
it explains the second equality in the computation below.

$$
\begin{aligned}
b_{i+1}=e s_{i}+s+\beta_{0}^{R}(N) & \geqslant(A+1) s_{i}+s \\
& =s_{i+1}+s_{i} \\
& \geqslant A b_{i}+A b_{i-1} \\
& \geqslant A b_{i}\left(1+\frac{1}{\ell(R)-1}\right)
\end{aligned}
$$

The penultimate inequality follows from (11) and the last one from [2, lem. 4.2.7].

(A.4) Rings with radical cube zero. Assume $\mathfrak{m}^{3}=0 \neq \mathfrak{m}^{2}$ and fix the notation:

$$
a=\operatorname{rank}_{\mathrm{k}} \mathfrak{m}^{2}, \quad e=\operatorname{rank}_{\mathfrak{k}} \mathfrak{m} / \mathfrak{m}^{2}, \quad \text { and } \quad r=\operatorname{rank}_{\mathrm{k}} \operatorname{Soc} R .
$$

Notice that the inclusion $\mathfrak{m}^{2} \subseteq \operatorname{Soc} R$ yields the inequality $a \leqslant r$.

Let $M$ be a finitely generated $R$-module, and set $b_{i}=\beta_{i}^{R}(M)$ for $i \geqslant 0$. We recall a few facts from Lescot's [19, §3]. There is an inequality

$$
b_{1} \geqslant e b_{0}-\operatorname{rank}_{\mathrm{k}}\left(\mathfrak{m} M / \mathfrak{m}^{2} M\right) \text {. }
$$

If $\mathrm{k}$ is not a direct summand of $M_{1}$, then equality holds; the converse is true if Soc $R=\mathfrak{m}^{2}$.

For every $i \geqslant 2$ there is an inequality

$$
b_{i} \geqslant e b_{i-1}-a b_{i-2} \text {. }
$$

If $\mathrm{k}$ is not a summand of $M_{1}$ and not a summand of $M_{2}$, then the equality

$$
b_{2}=e b_{1}-a b_{0}
$$

holds if and only if $\mathfrak{m}^{2} M=0$.

If $i \geqslant 3$, and $\mathrm{k}$ is not a summand of $M_{i}$ and not a summand of $M_{i-1}$, then

$$
b_{i}=e b_{i-1}-a b_{i-2} .
$$

If Soc $R=\mathfrak{m}^{2}$ and $\mathfrak{m}^{2} M=0$, then

$$
e b_{1} \geqslant r b_{0}+s(e-1)
$$

where $s$ is the k-rank of $M_{1}$.

The next result strengthens parts of Lescot's [19, thm. B].

(A.5) Theorem. Let $R$ be a local ring with $\mathfrak{m}^{3}=0 \neq \mathfrak{m}^{2}$ that is not Gorenstein. Let $M$ be a finitely generated $R$-module that satisfies $\beta_{1}^{R}(M)>\beta_{0}^{R}(M)$. With the notation from A.4.1 the following statements hold

(a) If $a \neq e$ or $a \neq r$, then the sequence $\left\{\beta_{i}^{R}(M)\right\}_{i \geqslant 0}$ is increasing and has termwise exponential growth.

(b) If $a=e=r$, then the sequence $\left\{\beta_{i}^{R}(M)\right\}_{i \geqslant 0}$ is non-decreasing with strict inequalities $\beta_{i}^{R}(M)>\beta_{i-1}^{R}(M)$ for all but, possibly, one index $i$. Moreover, if there is an equality $\beta_{j}^{R}(M)=\beta_{j-1}^{R}(M)$, then $j$ is at least 2 , and $\mathrm{k}$ is a direct summand of $M_{j+1}$ and not a direct summand of $M_{i}$ when $0 \leqslant i \leqslant j$.

Proof. For $i \geqslant 0$ set $b_{i}=\beta_{i}^{R}(M)$.

(a): First assume $a \neq r$, that is, $\mathfrak{m}^{2} \neq \operatorname{Soc} R$. By [19, prop. 3.9] and the assumption on $M$, the sequence $\left\{b_{i}\right\}_{i \geqslant 0}$ is increasing, and by Proposition (A.3) it has termwise exponential growth of rate $(r-a)(1+1 /(a+e))$.

Next, assume $a=r$. There are two cases to consider: 
Case $e<a$ : Since $\ell_{R}\left(R / \mathfrak{m}^{2}\right)-1=e$, it follows from Proposition A.2 and the assumption on $M$ that the sequence $\left\{b_{i}\right\}_{i \geqslant 0}$ is increasing and has termwise exponential growth of rate $a / e$.

Case $e>a$ : For every $i \geqslant 2$, the inequality (A.4.3) yields

$$
b_{i}-b_{i-1} \geqslant(e-1) b_{i-1}-a b_{i-2} \geqslant a\left(b_{i-1}-b_{i-2}\right) .
$$

By recursion, based on the assumption $b_{1}>b_{0}$, it follows that the sequence $\left\{b_{i}\right\}_{i \geqslant 0}$ is increasing. If $e=a+1$, then the sequence has termwise exponential growth by [21, prop. 3]. If $e>a+1$, then the same conclusion follows as (A.4.3) yields

$$
b_{i}>(e-a) b_{i-1} \text { for all } i \geqslant 2 \text {. }
$$

(b): Assume that $a=e=r$. First notice that if $i \geqslant 3$ and $\mathrm{k}$ is not a summand of $M_{i}$ and not a summand of $M_{i-1}$, then A.4.5 yields $b_{i-1}>b_{i-2}$, as $b_{i}>0$ by assumption. It follows that the sequence $\left\{b_{i}\right\}_{i \geqslant 0}$ is increasing if for every $i \geqslant 2$ the residue field $\mathrm{k}$ is not a direct summand of $M_{i}$.

Next, assume that $\mathrm{k}$ is a summand of a syzygy of $M$, and let $j$ be the least integer such that $M_{j+1}=\mathrm{k} \oplus N$ for some $R$-module $N$. The sequence $\left\{b_{i}\right\}_{i \geqslant j+1}$ is then increasing. Indeed, the sequence $\left\{\beta_{i}^{R}(\mathrm{k})\right\}_{i \geqslant 0}$ is increasing, as $R$ is not a hypersurface. If $j \geqslant 0$, then the sequence $\left\{\beta_{i}^{R}(N)\right\}_{i \geqslant 0}$ is non-decreasing by A.4.6), as $\mathfrak{m}^{2} N=0$. If $j=-1$, i.e. $\mathrm{k}$ is a summand of $M$, then the sequence $\left\{\beta_{i}^{R}(N)\right\}_{i \geqslant 1}$ is non-decreasing by (A.4.6), and $b_{1}>b_{0}$ by assumption. If $j=-1$ or $j=0$, it is thus immediate that the sequence $\left\{b_{i}\right\}_{i \geqslant 0}$ is increasing.

We can now assume that $j$ is at least 1 . In the next chain of inequalities, the right-most and left-most ones are already know; the inequalities in-between follow by application of (A.4.6) to the syzygies $M_{1}, \ldots, M_{j}$.

$$
\cdots>b_{j+1}>b_{j} \geqslant \cdots \geqslant b_{1}>b_{0}
$$

If $j=1$, it follows that the sequence $\left\{b_{i}\right\}_{i \geqslant 0}$ is increasing. If $j \geqslant 2$, then $b_{i-1}>b_{i-2}$ for $i$ with $j \geqslant i \geqslant 2$ by (A.4.5) and the assumption on $M$. In total, this gives inequalities $\cdots>b_{j+1}>b_{j} \geqslant b_{j-1}>\cdots>b_{1}>b_{0}$.

\section{ACKNOWLEDGMENTS}

We thank Luchezar L. Avramov and Sean Sather-Wagstaff for extensive comments on an earlier version of the paper. Thanks are also due to Christopher Monico and Roger W. Barnard for discussions related to the material in Section 6 A series of pertinent remarks from the anonymous referee helped us improve the exposition.

\section{REFERENCES}

1. David J. Anick, A counterexample to a conjecture of Serre, Ann. of Math. (2) 115 (1982), no. 1, 1-33. MR644015

2. Luchezar L. Avramov, Infinite free resolutions, Six lectures on commutative algebra (Bellaterra, 1996), Progr. Math., vol. 166, Birkhäuser, Basel, 1998, pp. 1-118. MR1648664

3. Luchezar L. Avramov, Srikanth Iyengar, and Claudia Miller, Homology over local homomorphisms, Amer. J. Math. 128 (2006), no. 1, 23-90. MR2197067

4. Luchezar L. Avramov and Jack Lescot, Bass numbers and Golod rings, Math. Scand. 51 (1982), no. 2, 199-211 (1983). MR690525

5. Luchezar L. Avramov and Oana Veliche, Stable cohomology over local rings, Adv. Math. 213 (2007), no. 1, 93-139. MR2331239

6. Jörgen Backelin and Ralf Fröberg, Poincaré series of short Artinian rings, J. Algebra 96 (1985), no. 2, 495-498. MR810542

7. Hyman Bass, Injective dimension in Noetherian rings, Trans. Amer. Math. Soc. 102 (1962), 18-29. MR0138644 
8. Hans-Bjørn Foxby, On the $\mu^{i}$ in a minimal injective resolution, Math. Scand. 29 (1971), 175-186 (1972). MR0309919

9. $\ldots$ On the $\mu^{i}$ in a minimal injective resolution II, Math. Scand. 41 (1977), no. 1, 19-44. MR0476801

10. Vesselin N. Gasharov and Irena V. Peeva, Boundedness versus periodicity over commutative local rings, Trans. Amer. Math. Soc. 320 (1990), no. 2, 569-580. MR967311

11. Daniel R. Grayson and Michael E. Stillman, Macaulay 2, a software system for research in algebraic geometry, available from http://www.math.uiuc.edu/Macaulay2/

12. Tor H. Gulliksen, On the deviations of a local ring, Math. Scand. 47 (1980), no. 1, 5-20. MR600076

13. Jürgen Herzog and Manfred Steurich, Two applications of change of rings theorems for Poincaré series, Proc. Amer. Math. Soc. 73 (1979), no. 2, 163-168. MR516457

14. Craig Huneke and Adela Vraciu, Rings that are almost Gorenstein, Pacific J. Math. 225 (2006), no. 1, 85-102. MR2233726

15. David A. Jorgensen and Graham J. Leuschke, On the growth of the Betti sequence of the canonical module, Math. Z. 256 (2007), no. 3, 647-659. MR2299575

16. Aleksei I. Kostrikin and Igor R. Šafarevič, Groups of homologies of nilpotent algebras, Dokl. Akad. Nauk SSSR (N.S.) 115 (1957), 1066-1069. MR0092776

17. Jack Lescot, La série de Bass d'un produit fibré d'anneaux locaux, C. R. Acad. Sci. Paris Sér. I Math. 293 (1981), no. 12, 569-571. MR647683

18. L La série de Bass d'un produit fibré d'anneaux locaux, Paul Dubreil and Marie-Paule Malliavin algebra seminar, 35th year (Paris, 1982), Lecture Notes in Math., vol. 1029, Springer, Berlin, 1983, pp. 218-239. MR732477

19. - Asymptotic properties of Betti numbers of modules over certain rings, J. Pure Appl. Algebra 38 (1985), no. 2-3, 287-298. MR814184

20. Gerson L. Levin and Luchezar L. Avramov, Factoring out the socle of a Gorenstein ring, J. Algebra 55 (1978), no. 1, 74-83. MR515760

21. Irena Peeva, Exponential growth of Betti numbers, J. Pure Appl. Algebra 126 (1998), no. 1-3, 317-323. MR1600558

22. Paul Roberts, Rings of type 1 are Gorenstein, Bull. London Math. Soc. 15 (1983), no. 1, 48-50. MR686348

23. Günter Scheja, Über die Bettizahlen lokaler Ringe, Math. Ann. 155 (1964), 155-172. MR0162819

24. Jean-Pierre Serre, Local algebra, Springer Monographs in Mathematics, Springer-Verlag, Berlin, 2000, Translated from the French by CheeWhye Chin and revised by the author. MR1771925

25. Li-Chuan Sun, Growth of Betti numbers of modules over local rings of small embedding codimension or small linkage number, J. Pure Appl. Algebra 96 (1994), no. 1, 57-71. MR1297441

26. William Teter, Rings which are a factor of a Gorenstein ring by its socle, Invent. Math. $\mathbf{2 3}$ (1974), 153-162. MR0366911

27. Wolmer V. Vasconcelos, Divisor theory in module categories, North-Holland Publishing Co., Amsterdam, 1974, North-Holland Mathematics Studies, No. 14, Notas de Matemática No. 53. [Notes on Mathematics, No. 53]. MR0498530

28. Hartmut Wiebe, Über homologische Invarianten lokaler Ringe, Math. Ann. 179 (1969), 257274. MR0255531

L. W. Christensen, Department of Mathematics and Statistics, Texas Tech UniverSiTy, LubBock, Texas 79409, U.S.A.

E-mail address: lars.w.christensen@ttu.edu

$U R L:$ http://www.math.ttu.edu/ lchriste

J. Striuli, Department of Mathematics and Computer Science, Fairfield University, FAirfield, Connecticut 06824, U.S.A.

E-mail address: jstriuli@fairfield.mail.edu

O. Veliche, Department of Mathematics, University of Utah, Salt Lake City, Utah 84112 , U.S.A.

E-mail address: oveliche@math.utah.edu

URL: http://www.math.utah.edu/ oveliche 